\title{
Chidamide in the treatment of peripheral T-cell lymphoma
}

This article was published in the following Dove Press journal:

OncoTargets and Therapy

12 January 2017

Number of times this article has been viewed

\section{Thomas S Chan \\ Eric Tse \\ Yok-Lam Kwong}

Department of Medicine, Queen

Mary Hospital, Hong Kong,

People's Republic of China
Correspondence: Yok-Lam Kwong Department of Medicine, Professorial Block, Queen Mary Hospital, Pokfulam Road, Hong Kong, People's Republic of China Tel +86 85222555859

Fax +86852 2974 I I 65 Email ylkwong@hkucc.hku.hk
Abstract: Mature T-cell lymphomas are aggressive malignancies. Treatment outcome is poor with conventional chemotherapy. They are about twice as common in Asia as compared with other non-Asian countries. Histone proteins form the basic structure of chromatin, and their acetylation at lysine residues relaxes chromatin structure, facilitating gene transcription. Conversely, histone deacetylation, catalyzed by histone deacetylases, compacts chromatin and represses gene transcription. Histone deacetylase inhibitors are an important class of antineoplastic agents. Chidamide is a novel orally active benzamide-type histone deacetylase inhibitor that has shown in vitro activities against a wide array of neoplasms. In Phase I trials, chidamide showed preferential efficacy in mature T-cell lymphomas. In a pivotal Phase II trial of chidamide in 79 patients with relapsed or refractory mature T-cell lymphomas, an overall response rate of $28 \%$ (complete remission/complete remission unconfirmed: $14 \%$ ) was achieved, with most responses occurring within the first 6 weeks of treatment. The median duration of response (DOR) was $9.9(1.1-40.8)$ months. Of 22 responders, 19 patients (86\%) had a DOR of $\geq 3$ months and eight patients ( $36 \%$ ) had a DOR of $>12$ months. Angioimmunoblastic T-cell lymphoma and anaplastic large cell lymphoma (anaplastic lymphoma kinase-negative) showed better response rates, with the most durable responses observed in angioimmunoblastic T-cell lymphoma patients. Safety profile was favorable, with very few cases of grade 3/4 toxicities observed. Chidamide is approved by the China Food and Drug Administration for the treatment of relapsed and refractory peripheral T-cell lymphomas.

Keywords: chidamide, peripheral T-cell lymphoma, benzamide, histone deacetylase inhibitors

\section{Mature T-cell lymphomas}

Mature T-cell lymphomas are a heterogeneous group of T-cell malignancies derived from postthymic mature $\mathrm{T}$ lymphocytes. There are geographical variations in their distribution. Mature T-cell lymphomas account for $\sim 5 \%-10 \%$ of lymphomas in Western countries. ${ }^{1}$ In Asian countries, however, they may account for up to $20 \%$ of all lymphomas. ${ }^{2}$ The classification of mature T-cell lymphomas relies on their predominant sites of involvement and clinical behavior. For nodal mature T-cell lymphomas, the most common subtypes include peripheral T-cell lymphoma, not otherwise specified, angioimmunoblastic T-cell lymphoma (AITL), and anaplastic large cell lymphoma (ALCL). For extranodal mature T-cell lymphomas, there is also a geographical variation in their incidences. In the West, the most common ones are cutaneous T-cell lymphomas, with mycosis fungoides occurring at the highest frequency. ${ }^{1}$ However, in Asia, extranodal NK/T-cell lymphoma, nasal type, is most frequent. ${ }^{2}$ Cutaneous T-cell lymphomas are very uncommon in Asian populations.

There is currently no standard chemotherapeutic regimen for nodal mature T-cell lymphomas. With the exception of anaplastic lymphoma kinase-positive ALCL and 
primary cutaneous ALCL, the response to conventional anthracycline-containing regimens remains poor. ${ }^{1}$ In a study involving 340 patients with peripheral T-cell lymphoma, not otherwise specified, the reported 5-year overall survival (OS) and failure-free survival were poor at $32 \%$ and $20 \%$, respectively. The addition of anthracycline did not seem to affect the outcome. ${ }^{3}$

Trials evaluating intensification of chemotherapy have resulted in variable success in prolonging event-free survival, ${ }^{4-6}$ but OS has remained unchanged. These results show that dose-intensified chemotherapy does not appear to be superior to conventional anthracycline-based regimens. Consequently, the use of high-dose chemotherapy and autologous hematopoietic stem cell transplantation has also been advocated as a consolidation strategy for patients who achieve a complete remission (CR). ${ }^{7,8}$ Improvement in progression-free survival (PFS) and OS is achievable, but the strategy is limited to patients who are in CR after initial chemotherapy. However, many patients are ineligible for hematopoietic stem cell transplantation, because of age or failure to achieve remission.

Given the poor results with cytotoxic chemotherapy, there is a pressing need for the development of novel treatment strategies for mature T-cell lymphomas. ${ }^{9}$

\section{Epigenetics, histone deacetylase, and cancer}

Carcinogenesis can be due to genetic mutations that change DNA sequences. The results are either increased expression of oncogenes or decreased expression of tumor suppressor genes. However, gene expression can also be affected by other pathogenetic mechanisms. Epigenetic modifications are defined as heritable changes in gene expression that are not due to alterations in DNA sequence. ${ }^{10}$ There are two major epigenetic processes in cancer, ${ }^{11}$ DNA hypermethylation and posttranslational histone modification.

Methylation of DNA occurs at sites where there are cytosines preceding guanines (CpGs). These CpGs are distributed nonrandomly and are particularly concentrated as islands in close proximity to gene promoters. Methylation of the cytosine residuals in these $\mathrm{CpG}$ islands in general results in suppression of gene expression. Hence, hypermethylation of $\mathrm{CpG}$ islands in the promoters of tumor suppressor genes constitutes an important pathogenetic mechanism in many cancers. Examples of tumor suppressor genes inactivated by promoter DNA hypermethylation include $R B,{ }^{12} P 16,{ }^{13}$ and $B R C A 1 .^{14}$

Histone proteins form the basic repeating units of chromatin and provide a spool for DNA to wrap around. ${ }^{15,16}$
The amino terminal tails of histone proteins protrude out of the nucleosomes. The acetylation of histones is an epigenetic phenomenon that modulates gene transcription. Acetylation of the $\varepsilon$-amino group of lysine residues in the amino terminal tails of histone proteins, catalyzed by histone acetyltransferases, neutralizes their positive charges, resulting in relaxation of chromatin structure, thereby allowing better access of transcription factors to their target genes. ${ }^{15}$ Acetylated histones also provide binding sites for bromodomain proteins, which are transcriptional activators. On the other hand, the removal of an acetyl group from lysine residues, catalyzed by histone deacetylases (HDACs), results in chromatin condensation and hence repression of gene transcription. ${ }^{15}$ HDACs play important roles in the control of gene transcription and affect multiple cellular processes. ${ }^{16}$

\section{HDAC}

Classical HDACs can be categorized into four classes, I, IIa, IIb, and IV; according to their homology with their corresponding yeast proteins. ${ }^{16}$ They are zinc-dependent proteases with very high deacetylase activities for substrates. ${ }^{15}$ Class I HDACs are localized mainly to the nucleus and are found ubiquitously in different tissues and organs. Deletion of any of them is lethal in mice, showing that they are nonredundant, with each controlling specific cellular processes. ${ }^{15}$ Class II HDACs shuttle in and out of the nucleus and are more restricted in their tissue distribution. Another type of HDAC, often called class III HDACs, are NAD-dependent proteins. Referred also to as sirtuins, class III HDACs (sirtuins 1-7) are implicated in influencing a broad range of processes other than control of gene transcription, including apoptosis, aging, and inflammation. ${ }^{15}$

HDACs play important roles in oncogenesis. Class I HDACs inhibit apoptosis and promote cellular proliferation. Class II HDACs may enhance angiogenesis. ${ }^{15,16}$ Hence, inhibition of HDAC is a valid strategy in cancer therapy.

\section{HDAC inhibitors}

Classical HDACs are zinc dependent. HDAC inhibitors (HDACis), therefore, typically contain a moiety that occupies the catalytic core of the zinc-binding site, thereby interfering with zinc binding. Hence, HDACi targeting class I and II HDACs has a general structure consisting of a zinc-chelating moiety, a linker, and an external surface recognition motif. Depending on the zinc-chelating moieties, HDACi can be divided into two broad categories, hydroxamic acid derivatives and nonhydroxamic acid derivatives. Three types of nonhydroxamic acid derivatives can be distinguished: thiol and thiol derivatives, benzamides, and ketones (Table 1). ${ }^{16}$ 
Table I Types of histone deacetylase inhibitors and their current approval status in the treatment of mature T-cell lymphomas

\begin{tabular}{|c|c|c|}
\hline Type & Examples & $\begin{array}{l}\text { Regulatory approval } \\
\text { for T-cell lymphoma }\end{array}$ \\
\hline \multirow[t]{3}{*}{ Hydroxamic acids } & Vorinostat & Yes (US FDA) \\
\hline & Belinostat & Yes (US FDA) \\
\hline & Panobinostat & No \\
\hline \multirow[t]{2}{*}{ Short-chain aliphatic acids } & Phenylbutyrate & No \\
\hline & Valproic acid & No \\
\hline Cyclic tetrapeptides & Romidepsin & Yes (US FDA) \\
\hline Benzamides & Chidamide & Yes (China FDA) \\
\hline
\end{tabular}

Abbreviation: FDA, Food and Drug Administration.

\section{Chidamide}

Chidamide is an orally active novel benzamide-type HDACi, designed with molecular docking analysis employing HDAC-like protein and quantitative structure-activity relationship studies. ${ }^{17}$ It has a high affinity for class I HDAC (IC $_{50}$ for HDAC1: $0.095 \mu \mathrm{M}$; HDAC2: $0.16 \mu \mathrm{M}$; and HDAC3: $0.067 \mu \mathrm{M})$ and class IIb HDAC ( IC $_{50}$ for HDAC10: $0.075 \mu \mathrm{M}) .{ }^{17} \mathrm{It}$ has minimal inhibitory activity against other class I, IIa, and IV HDACs. Chidamide treatment leads to increased acetylation of histone $\mathrm{H} 3$ at Lys9/Lys18 and $\mathrm{H} 4$ at Lys8, resulting ultimately in activation of gene transcription. ${ }^{17}$

\section{Preclinical studies of chidamide}

Chidamide has been tested extensively for its tumor inhibitory activity. In colonic cancer cell lines, chidamide inhibited the PI3K/AKT and RAS/MAPK signaling pathways, leading to cell cycle arrest and hence suppression of proliferation. ${ }^{18}$ In leukemia lines, chidamide induced caspase-dependent apoptosis through generation of reactive oxygen species, resulting in mitochondrial dysfunction and cytochrome c release. ${ }^{19}$ In hepatocellular carcinoma cells, chidamide upregulated the cyclin-dependent kinase inhibitor P21, leading to cell cycle arrest. ${ }^{20}$ In pancreatic cancer cell lines and in vivo tumors, chidamide treatment upregulated the proapoptotic BAX/BCL2 expression ratio and suppressed cellular proliferation by promoting mitochondrial pathwaydependent cell apoptosis. ${ }^{21}$

Chidamide may also synergize with chemotherapeutic agents. In pancreatic cancer cell lines, chidamide enhanced gemcitabine-induced cell growth arrest and apoptosis in a synergistic manner, which was due to downregulation of the antiapoptotic protein MCL-1 and loss of mitochondrial membrane potential. Chidamide also increased gemcitabine-induced DNA double-strand breaks and $\mathrm{S}$ phase arrest and suppressed CHK1 expression, thereby abrogating the G2/M cell cycle checkpoint. ${ }^{22}$ In non-small-cell lung cancer cell lines, chidamide synergized with carboplatin in suppressing cellular proliferation. ${ }^{23}$ There was increase in carboplatin-induced apoptosis, as measured by mitochondrial membrane potential and cleaved PARP1 levels. Combination with other platinum drugs including cisplatin and oxaliplatin showed similar effects. ${ }^{23}$ In a murine xenograft model of colonic cancer, chidamide combined with 5-fluorouracil increased P53, phosphorylated-P53, and P21 levels, but suppressed cyclin-dependent kinase 4 expression in tumor cells. The combination also blocked signaling via the AKT, mTOR, RAF, and ERK1/2 pathways. $^{24}$

Chidamide may also modulate antitumor immune mechanisms. The ex vivo cytotoxic effects of peripheral blood mononuclear cells, particularly natural killer cells, on target leukemia cell lines were increased by chidamide. ${ }^{17}$ Treatment of myeloid leukemia cells (primary samples and cell lines) with chidamide led to increased expression of the potentially immunogenic antigen preferentially expressed antigen of melanoma, which was further enhanced by the demethylating agent decitabine. ${ }^{25}$ Accordingly, pretreatment of leukemia cell line cells with chidamide and/or decitabine increased their sensitivity to purified cytotoxic T-cells that recognized preferentially expressed antigen of melanoma. ${ }^{25}$ These results suggest that chidamide treatment may also target neoplastic cells indirectly via immune mechanisms.

\section{Phase I study of chidamide in patients with advanced solid tumors and lymphomas}

The Phase I study was designed to recruit patients with advanced solid tumors or lymphomas that were refractory to standard treatment. ${ }^{26}$ Chidamide used as a single agent was administered orally in sequential cohorts of $5 \mathrm{mg}, 10 \mathrm{mg}$, $17.5 \mathrm{mg}, 25 \mathrm{mg}, 32.5 \mathrm{mg}$, or $50 \mathrm{mg}$, either twice (biw) or three times (tiw) per week for 4 consecutive weeks every 6 weeks.

Thirty-one patients were enrolled. At $50 \mathrm{mg}$ biw, two of four patients developed grade 3 hematologic toxicities. At $50 \mathrm{mg}$ tiw, two patients experienced dose-limiting adverse events (grade 3 diarrhea, grade 3 nausea, and vomiting). At $32.5 \mathrm{mg}$ tiw, four of seven patients experienced grade 3 hematologic adverse events. Therefore, the maximum tolerated dose for the biw dosing schedule was set at $50 \mathrm{mg}$ and for the tiw dosing schedule at $32.5 \mathrm{mg}$.

Pharmacokinetic studies showed that plasma chidamide concentrations peaked in the majority of patients within 0.5-2 hours of drug administration and returned to baseline levels in 48 hours. Elimination half-life ranged from 16 hours to 18 hours.

Adverse events are shown in Table 2. In general, chidamide appeared to be very well tolerated, and significant 
Table 2 Adverse events of chidamide in $>5 \%$ of patients in clinical trials

\begin{tabular}{|c|c|c|c|c|c|c|}
\hline Adverse events & $\begin{array}{l}\text { Total } \\
\text { number }\end{array}$ & Percentage & $\begin{array}{l}\text { Grade } 3 \\
\text { number }\end{array}$ & Percentage & $\begin{array}{l}\text { Grade } 4 \\
\text { number }\end{array}$ & Percentage \\
\hline \multicolumn{7}{|l|}{ Phase I $(\mathrm{N}=3 \mathrm{I})^{\mathrm{a}}$} \\
\hline Fatigue & 11 & 35 & 0 & 0 & 0 & 0 \\
\hline Thrombocytopenia & 8 & 26 & 2 & 6 & 0 & 0 \\
\hline Anorexia & 8 & 26 & 2 & 6 & 0 & 0 \\
\hline Leukopenia & 7 & 23 & 3 & 10 & 0 & 0 \\
\hline Anemia & 6 & 19 & 0 & 0 & 0 & 0 \\
\hline Nausea & 5 & 16 & 0 & 0 & 0 & 0 \\
\hline Diarrhea & 5 & 16 & I & 3 & 0 & 0 \\
\hline Dizziness & 4 & 13 & 0 & 0 & 0 & 0 \\
\hline Vomiting & 2 & 6 & I & 3 & 0 & 0 \\
\hline Flatulence & 2 & 6 & 0 & 0 & 0 & 0 \\
\hline Hemoptysis & 2 & 6 & 0 & 0 & 0 & 0 \\
\hline Insomnia & 2 & 6 & 0 & 0 & 0 & 0 \\
\hline Headache & 2 & 6 & 0 & 0 & 0 & 0 \\
\hline \multicolumn{7}{|l|}{ Phase II $(\mathrm{N}=83)^{\mathrm{b}}$} \\
\hline Thrombocytopenia & 42 & 51 & 13 & 16 & 5 & 6 \\
\hline Leukopenia & 33 & 40 & 10 & 12 & I & I \\
\hline Neutropenia & 18 & 22 & 7 & 8 & 2 & 2 \\
\hline Prolonged QTc interval & II & 13 & I & 13 & 0 & 0 \\
\hline Fatigue & 8 & 10 & 0 & 0 & 0 & 0 \\
\hline Anemia & 7 & 8 & 3 & 4 & I & 1 \\
\hline Decreased appetite & 7 & 8 & 2 & 2 & 0 & 0 \\
\hline Fever & 7 & 8 & 0 & 0 & 0 & 0 \\
\hline Nausea & 7 & 8 & 0 & 0 & 0 & 0 \\
\hline Diarrhea & 7 & 8 & 0 & 0 & 0 & 0 \\
\hline Pericardial effusion & 6 & 7 & 0 & 0 & 0 & 0 \\
\hline Increased ALT & 6 & 7 & I & 1 & 0 & 0 \\
\hline Increase GGT & 5 & 6 & I & 1 & 0 & 0 \\
\hline Increased CPK & 5 & 6 & 0 & 0 & 0 & 0 \\
\hline Lung infection & 5 & 6 & I & 1 & 0 & 0 \\
\hline
\end{tabular}

Notes: aln patients with solid tumors and different lymphomas. Data from a previous study. ${ }^{26}$ bln patients with T-cell lymphomas. Reproduced and adapted, with permission, from Y Shi et al. Results From A Multicenter, Open-Label, Pivotal Phase II Study Of Chidamide In Relapsed Or Refractory Peripheral T-Cell Lymphoma. Annals of Oncology. 2015;26 (8):1766-177I. ${ }^{27}$ Published by Oxford University Press on behalf of the European Society for Medical Oncology online at: http://annonc.oxfordjournals.org/ content/26/8/I766.abstract. Published under a Standard License only. For permissions please email: journals.permissions@oup.com.

Abbreviations: N, number of patients; ALT, alanine aminotransferase; GGT, gamma-glutamyl transferase; CPK, creatine phosphokinase.

toxicity ( $>$ grade 3 ) was only observed in the $32.5 \mathrm{mg}$ tiw and the $50 \mathrm{mg}$ tiw cohorts.

Twenty-five patients were evaluable. The best response was partial remission in five patients and stable disease in eleven patients. Four of the five patients with partial remission had mature T-cell lymphoma (Table 3). These observations provided the basis for testing chidamide in mature T-cell lymphoma.

\section{Pivotal Phase II study of chidamide in relapsed or refractory peripheral T-cell lymphoma}

The pivotal Phase II study was an open-label, single-arm, multicenter investigation of chidamide monotherapy in peripheral T-cell lymphomas that had relapsed from or were refractory to one or more systemic therapies. ${ }^{27}$ Final eligibility required central pathology review for confirmation of diagnosis. Patients received chidamide at $30 \mathrm{mg}$ biw until disease progression, unacceptable toxicity, or patient/ investigator withdrawal. The primary end point was overall response rate. Secondary end points included time to response, duration of response (DOR), PFS, proportion of patients with DOR $\geq 12$ weeks, OS, and profile of adverse events. Efficacy was assessed every 6 weeks, with results verified by an independent review committee.

Seventy-nine patients were evaluable. The overall response rate was $28 \%(\mathrm{CR} / \mathrm{CR}$ unconfirmed: $14 \%$; partial remission: 14\%). Most responses occurred within the first 6 weeks of treatment, although some responses occurred at as late as 18 weeks. The median DOR was $9.9(1.1-40.8)$ months. Of the 22 responders, 19 patients $(86 \%)$ had a DOR of $\geq 3$ months, and eight patients $(36 \%)$ had a DOR of $>12$ months. No specific factors impacted on outcome, although AITL and anaplastic lymphoma kinase-negative ALCL appeared to 
Table 3 Response of mature T-cell lymphomas to chidamide in clinical trials

\begin{tabular}{|c|c|c|c|c|c|}
\hline Pathology & $\begin{array}{l}\text { Number } \\
\text { of cases }\end{array}$ & $\begin{array}{l}\text { ORR } \\
\text { number }\end{array}$ & Percentage & $\begin{array}{l}\text { CR/CR } \\
\text { unconfirmed } \\
\text { number }\end{array}$ & Percentage \\
\hline \multicolumn{6}{|l|}{ Phase $\mathrm{I}^{\mathrm{a}}$} \\
\hline CTCL & NA & I & NA & 0 & 0 \\
\hline SPTCL & NA & I & NA & 0 & 0 \\
\hline PTCL-NOS & NA & I & NA & 0 & 0 \\
\hline $\mathrm{ALCL}$ & NA & I & NA & 0 & 0 \\
\hline \multicolumn{6}{|l|}{ Phase II } \\
\hline AITL & 10 & 5 & 50 & 4 & 40 \\
\hline \multicolumn{6}{|l|}{$\mathrm{ALCL}$} \\
\hline ALK+ & 6 & 2 & 33 & 0 & 0 \\
\hline ALK- & 11 & 5 & 45 & 4 & 36 \\
\hline PTCL-NOS & 27 & 6 & 22 & 2 & 7 \\
\hline ENKL & 16 & 3 & 19 & 1 & 6 \\
\hline Others & 9 & I & 11 & 0 & 0 \\
\hline
\end{tabular}

Notes: Data from Dong et $\mathrm{al}^{26}$ (Phase I trial) and Shi et al ${ }^{27}$ (Phase II trial). ${ }^{\mathrm{a}} \mathrm{A}$ total of six cases of mature T-cell lymphomas were included in this trial.

Abbreviations: ORR, overall response rate; CR, complete remission; CTCL, cutaneous T-cell lymphoma; SPTCL, subcutaneous panniculitis-like T-cell lymphoma; PTCLNOS, peripheral T-cell lymphoma, not otherwise specified; ALCL, anaplastic large cell lymphoma; AITL, angioimmunoblastic T-cell lymphoma; ALK, anaplastic lymphoma kinase; ENKL, extranodal NK/T-cell lymphoma; NA, not available.

have more favorable response rates (Table 3). In fact, the most durable responses were seen in three patients with AITL. The median PFS was $2.1(0.3-44.9)$ months, and the median OS was $21.4(0.3-50.1)$ months.

Adverse events occurred in $82 \%$ of patients (Table 2). Those that occurred in $\geq 10 \%$ patients were thrombocytopenia (51\%), leukopenia (40\%), neutropenia (22\%), and fatigue (10\%). Adverse events of $\geq$ grade 3 were thrombocytopenia (22\%), leukopenia (13\%), and neutropenia (11\%). Hematologic toxicities occurred mostly in the first 6 weeks of treatment. These adverse events led to drug discontinuation in 14 patients $(17 \%)$ and dose reduction in six patients (7\%). Prolongation of QTc interval was observed in eleven patients (13\%).

These results indicate that chidamide has significant activity against mature T-cell lymphomas. Its efficacy is similar to those observed in two other HDACis, romidepsin ${ }^{28}$ and belinostat. ${ }^{29}$ A recent study of romidepsin treatment of Epstein-Barr virus (EBV)-positive NK/T-cell lymphoma resulted in fatal EBV reactivation in some cases, suggesting that histone deacetylation might contribute to suppression of EBV proliferation in infected tumor cells. ${ }^{30}$ Hence, care should be exercised in the use of chidamide and other HDACi in EBV-positive lymphomas. Among the currently available HDACi, chidamide has the advantage of being an oral medication. It was approved by the China Food and Drug Administration for the treatment of relapsed and refractory peripheral T-cell lymphoma in December 2014.

\section{Ongoing studies of chidamide}

Chidamide is now undergoing clinical trials in Asia and North America, predominantly in lymphoma patients. Since the number of patients with cutaneous T-cell lymphoma treated with chidamide was small, the testing of this drug in such patients will be of interest. Finally, it remains to be defined if chidamide in combination with chemotherapy may be more effective than its use as single agent.

\section{Conclusion}

Chidamide is a novel HDACi that has demonstrated significant therapeutic efficacy in peripheral T-cell lymphomas, which is achieved with a very favorable toxicity profile. Future studies should define if combination of chidamide with other targeted drugs or chemotherapy may have increased efficacy in peripheral T-cell lymphomas, the treatment of which remains a challenge.

\section{Disclosure}

The authors report no conflicts of interest in this work.

\section{References}

1. Vose J, Armitage J, Weisenburger D; International T-Cell Lymphoma Project. International peripheral T-cell and natural killer/T-cell lymphoma study: pathology findings and clinical outcomes. J Clin Oncol. 2008;26(25):4124-4130.

2. Au WY, Ma SY, Chim CS, et al. Clinicopathologic features and treatment outcome of mature T-cell and natural killer-cell lymphomas diagnosed according to the World Health Organization classification scheme: a single center experience of 10 years. Ann Oncol. 2005;16(2): 206-214.

3. Weisenburger DD, Savage KJ, Harris NL, et al; International Peripheral T-Cell Lymphoma Project. Peripheral T-cell lymphoma, not otherwise specified: a report of 340 cases from the International Peripheral T-cell lymphoma project. Blood. 2011;117(12):3402-3408.

4. Simon A, Peoch M, Casassus P, et al. Upfront VIP-reinforced-ABVD (VIP-rABVD) is not superior to $\mathrm{CHOP} / 21$ in newly diagnosed peripheral T cell lymphoma. Results of the randomized phase III trial GOELAMSLTP95. Br J Haematol. 2010;151(2):159-166. 
5. Schmitz N, Trümper L, Ziepert M, et al. Treatment and prognosis of mature T-cell and NK-cell lymphoma: an analysis of patients with T-cell lymphoma treated in studies of the German High-Grade Non-Hodgkin Lymphoma Study Group. Blood. 2010;116(18):3418-3425.

6. Abramson JS, Feldman T, Kroll-Desrosiers AR, et al. Peripheral T-cell lymphomas in a large US multicenter cohort: prognostication in the modern era including impact of frontline therapy. Ann Oncol. 2014; 25(11):2211-2217.

7. Reimer P, Rüdiger T, Geissinger E, et al. Autologous stem-cell transplantation as first-line therapy in peripheral T-cell lymphomas: results of a prospective multicenter study. J Clin Oncol. 2009;27(1):106-113.

8. d'Amore F, Relander T, Lauritzsen GF, et al. Up-front autologous stem-cell transplantation in peripheral T-cell lymphoma: NLG-T-01. J Clin Oncol. 2012;30(25):3093-3099.

9. Chan TS, Kwong YL, Tse E. Novel therapeutic agents for T-cell lymphomas. Discov Med. 2013;16(86):27-35.

10. Holliday R. The inheritance of epigenetic defects. Science. 1987; 238(4824):163-170.

11. Esteller M. Epigenetics in cancer. $N$ Engl J Med. 2008;358(11): 1148-1159.

12. Greger V, Passarge E, Hopping W, Messmer E, Horsthemke B. Epigenetic changes may contribute to the formation and spontaneous regression of retinoblastoma. Hum Genet. 1989;83(2):155-158.

13. Merlo A, Herman JG, Mao L, et al. $5^{\prime} \mathrm{CpG}$ island methylation is associated with transcriptional silencing of the tumour suppressor p16/ CDKN2/MTS1 in human cancers. Nat Med. 1995;1(7):686-692.

14. Esteller M, Silva JM, Dominguez G, et al. Promoter hypermethylation and BRCA1 inactivation in sporadic breast and ovarian tumors. $J$ Natl Cancer Inst. 2000;92(7):564-569.

15. Haberland M, Montgomery RL, Olson EN. The many roles of histone deacetylases in development and physiology: implications for disease and therapy. Nat Rev Genet. 2009;10(1):32-42.

16. Paris M, Porcelloni M, Binaschi M, Fattori D. Histone deacetylase inhibitors: from bench to clinic. J Med Chem. 2008;51(6):1505-1529.

17. Ning ZQ, Li ZB, Newman MJ, et al. Chidamide (CS055/HBI-8000): a new histone deacetylase inhibitor of the benzamide class with antitumor activity and the ability to enhance immune cell-mediated tumor cell cytotoxicity. Cancer Chemother Pharmacol. 2012;69(4):901-909.

18. Liu L, Chen B, Qin S, et al. A novel histone deacetylase inhibitor chidamide induces apoptosis of human colon cancer cells. Biochem Biophys Res Commun. 2010;392(2):190-195.

19. Gong K, Xie J, Yi H, Li W. CS055 (Chidamide/HBI-8000), a novel histone deacetylase inhibitor, induces G1 arrest, ROS-dependent apoptosis and differentiation in human leukaemia cells. Biochem J. 2012;443(3): 735-746.
20. Wang H, Guo Y, Fu M, et al. Antitumor activity of chidamide in hepatocellular carcinoma cell lines. Mol Med Rep. 2012;5(6):1503-1508.

21. Zhao B, He T. Chidamide, a histone deacetylase inhibitor, functions as a tumor inhibitor by modulating the ratio of Bax/Bcl-2 and P21 in pancreatic cancer. Oncol Rep. 2015;33(1):304-310.

22. Qiao Z, Ren S, Li W, et al. Chidamide, a novel histone deacetylase inhibitor, synergistically enhances gemcitabine cytotoxicity in pancreatic cancer cells. Biochem Biophys Res Commun. 2013;434(1):95-101.

23. Zhou Y, Pan DS, Shan S, et al. Non-toxic dose chidamide synergistically enhances platinum-induced DNA damage responses and apoptosis in non-small-cell lung cancer cells. Biomed Pharmacother. 2014;68(4): 483-491.

24. Liu L, Qiu S, Liu Y, et al. Chidamide and 5-flurouracil show a synergistic antitumor effect on human colon cancer xenografts in nude mice. Neoplasma. 2016;63(2):193-200.

25. Yao Y, Zhou J, Wang L, et al. Increased PRAME-specific CTL killing of acute myeloid leukemia cells by either a novel histone deacetylase inhibitor chidamide alone or combined treatment with decitabine. PLoS One. 2013;8(8):e70522.

26. Dong M, Ning ZQ, Xing PY, et al. Phase I study of chidamide (CS055/ HBI-8000), a new histone deacetylase inhibitor, in patients with advanced solid tumors and lymphomas. Cancer Chemother Pharmacol. 2012; 69(6):1413-1422.

27. Shi Y, Dong M, Hong X, et al. Results from a multicenter, open-label, pivotal phase II study of chidamide in relapsed or refractory peripheral T-cell lymphoma. Ann Oncol. 2015;26(8):1766-1771.

28. Coiffier B, Pro B, Prince HM, et al. Results from a pivotal, openlabel, phase II study of romidepsin in relapsed or refractory peripheral T-cell lymphoma after prior systemic therapy. J Clin Oncol. 2012; 30(6):631-636.

29. O'Connor OA, Horwitz S, Masszi T, et al. Belinostat in patients with relapsed or refractory peripheral T-cell lymphoma: results of the pivotal phase II BELIEF (CLN-19) study. J Clin Oncol. 2015;33(23): 2492-2499.

30. Kim SJ, Kim JH, Ki CS, Ko YH, Kim JS, Kim WS. Epstein-Barr virus reactivation in extranodal natural killer/T-cell lymphoma patients: a previously unrecognized serious adverse event in a pilot study with romidepsin. Ann Oncol. 2016;27(3):508-513.
OncoTargets and Therapy

\section{Publish your work in this journal}

OncoTargets and Therapy is an international, peer-reviewed, open access journal focusing on the pathological basis of all cancers, potential targets for therapy and treatment protocols employed to improve the management of cancer patients. The journal also focuses on the impact of management programs and new therapeutic agents and protocols on

\section{Dovepress}

patient perspectives such as quality of life, adherence and satisfaction The manuscript management system is completely online and includes a very quick and fair peer-review system, which is all easy to use. Visit http://www.dovepress.com/testimonials.php to read real quotes from published authors. 